\title{
Does the incidence, severity, or case fatality of stroke vary in southern England?
}

\author{
C D A Wolfe, N A Taub, J Woodrow, E Richardson, F G Warburton, P G J Burney
}

\begin{abstract}
Study objectives-To determine differences in incidence and case fatality of stroke in district health authorities with differing standardised mortality ratios (SMR) for stroke in residents aged under 65 years in whom death from stroke is considered 'avoidable'.

Design-Registration of first ever strokes in three district health authorities. Patients were assessed and followed up over one year by one of three observers.

Setting-West Lambeth, Lewisham and North Southwark, and Tunbridge Wells District Health Authorities in south east England.

Participants-Patients under the age of 75 years having a first ever in a lifetime stroke between 15 August 1989 and 14 August 1990. Measurements and main results-Age specific incidence rates and survival time from stroke to death. Severity was assessed in terms of the level of consciousness and the presence of speech, urinary, and motor impairment within the first 24 hours of the stroke.
\end{abstract}

Altogether 386 strokes were registered. There was a significant difference in the incidence rate between district health authorities in those aged under $65(p<0 \cdot 01)$. The overall case fatality was $26 \%$ at three weeks with no significant difference between the districts. Poor survival was associated jointly with increased age and with coma, incontinence, and swallowing impairment in the first $\mathbf{2 4}$ hours after a stroke.

Conclusions-The SMRs for stroke in those aged under 65 in these three health districts reflect the incidence of stroke. Case fatality at three weeks does not vary between these districts and consequently would not be a sensitive indicator of the quality of care. This also suggests that differences in services between the districts did not lead to changes in prognosis. In districts with high SMRs for stroke there is a need for further study and reduction of risk factors, thereby reducing the incidence and burden of stroke locally. This study provides a framework for assessing the needs for stroke prevention and treatment in both rural and urban areas without an elaborate protocol and detailed neurological assessment.

f Epidemiol Community Health 1993; 47: 139-143

Stroke is one of the commonest causes of physical disability and death in adults ${ }^{12}$ but for someone less than 65 years to suffer a stroke and die as a result of it is considered 'avoidable'. ${ }^{3}{ }^{4}$ Death is considered avoidable because there are effective means of monitoring and controlling blood pressure in most patients. There are noticable differences in the standardised mortality ratios (SMRs) for stroke between countries ${ }^{5}$ and between regions in the same country. ${ }^{4-7}$ Malmgren $e t ~ a l^{8}$ reviewed 65 stroke incidence studies, of which only one in the UK was considered ideal. This was the Oxfordshire Community Stroke Project, ${ }^{9}$ which was based on selected general practices and reflects the incidence in a predominantly rural setting in the mid 1980s. The SMR for hypertension and stroke in district health authorities of the South East Thames Regional Health Authority, in the south east of England ranges from 70 to $134^{10}$ in residents aged under 65 (England and Wales=100). This study aims to ascertain whether differences in these SMRs in those aged under 65 years, in whom death is considered avoidable, are the result of differences in incidence or case fatality, or both. The study is not limited to those patients aged under 65 years but includes those in the age group 65 to 74 , in whom $25 \%$ of all strokes occur, and consequently represents about half the incidence cases of stroke in the study areas.

The study, unlike previous studies in the United Kingdom, is based on district health authority populations rather than selected general practices and has been carried out by public health physicians who need to be able to interpret the SMRs for such diseases as stroke and make policy on preventive strategies as a result of these investigations.

\section{Methods}

Stroke registers were set up in three districts of the South East Thames Regional Health AuthorityWest Lambeth (WL) and Lewisham and North Southwark (LNS) are inner London districts and Tunbridge Wells (TW) is a rural district 40 miles from London. WL has a high SMR (134: based on 366 deaths), LNS a moderate SMR (108: based on 647 deaths), and TW a low SMR (70: based on 264 deaths) for hypertension and cerebrovascular disease in those aged under 65 years. ${ }^{10}$ The denominators for the incidence rates were the Office of Population Censuses and Surveys 1988 mid-year estimates. ${ }^{14}$ The total study population under the age of 75 years was 621966 , with 148034 in WL, 293364 in LNS, and 180568 in TW. To detect a significant difference in case fatality rates at three weeks at the $5 \%$ level with a power of 0.8 when the true rates are $20 \%$ and $40 \%$, at least 85 strokes would be required in each 
of two districts. Similarly, to detect a difference in crude incidence rates when the true rates are 1.75 and $2 \cdot 25$ per thousand, populations of at least 130000 are required in each district. Both these conditions were met by studying the three districts over a one year period. The register was piloted in WL for six months before this.

\section{SOURCES OF NOTIFICATION}

All general practitioners (GPs) with patients living in the three districts were contacted, sent notification booklets to enable them to register a patient with minimum inconvenience, and invited to a seminar to discuss the project. A bi-monthly newsletter was sent to each GP. This helped to maintain cooperation and also asked for further notification of new cases. A letter was sent to the GP after each case was detected and all GPs were phoned and visited once during the study period. A questionnaire was also sent at the end of the year asking about further cases not previously reported. Similar notification systems were arranged with the district nursing and rehabilitation services, which were contacted on a weekly basis, and consultant physicians, who were sent the newsletter. Three fieldworkers identified hospital patients by looking through casualty admission registers and visiting wards on a weekly basis. Hospital pathology and coroners' post mortem reports and district residents' death certificates were all inspected. The fieldworkers visited all hospitals in the districts and also, in London, the hospitals in the surrounding area to which residents of these districts were known to have been admitted in the past after strokes. Consequently, 28 hospitals were involved in the study.

\section{CRITERIA FOR REGISTRATION}

The WHO definition of a stroke was used for all cases of first ever in a lifetime stroke under the age of 75 years whether or not they survived the event. ${ }^{11}$ Details of community cases and sudden deaths were also obtained from GP records. All questionnaires were checked by $\mathrm{CW}$ to ensure entry criteria were fulfilled, and if the diagnosis was in doubt there was further discussion with the relevant clinicians before deciding on whether to include a case.

Information was collected from either the patient, the hospital or GP notes, health care workers, or relatives. Records were made of the date of stroke, demographic details, and an

Table I Notifications from each source. Numbers (\%)*

\begin{tabular}{lcccc}
\hline Notification source & $\begin{array}{l}\text { West } \\
\text { Lambeth }\end{array}$ & $\begin{array}{l}\text { Lewisham } \mathbb{E} \\
\text { N Southwark }\end{array}$ & $\begin{array}{c}\text { Tunbridge } \\
\text { Wells }\end{array}$ & Total \\
\hline General practitioner & $19(19)$ & $30(17)$ & $18(17)$ & $67(17)$ \\
District nurses & $6(6)$ & $4(2)$ & $2(2)$ & $12(3)$ \\
Rehabilitation services & $21(21)$ & $17(9)$ & $3(3)$ & $41(11)$ \\
Hospital staff & $2(2)$ & $12(7)$ & $2(2)$ & $16(4)$ \\
Death certificates/coroner & $17(17)$ & $31(17)$ & $14(13)$ & $62(16)$ \\
Register/fieldworker & $53(54)$ & $99(55)$ & $70(65)$ & $222(58)$ \\
Others & $4(4)$ & $1(1)$ & $1(1)$ & $6(2)$ \\
\hline *Percentages do not sum to & 100 because some patients were notified more than once &
\end{tabular}

assessment of the patient's activities of daily living (ADL) before stroke using the Barthel 20 point scale $^{12}$ and the Rankin 6 point scale for handicap. ${ }^{13}$ Clinical details of maximal impairment within the first 24 hours included the level of consciousness, paralysis, speech, and urinary or swallowing impairment. For cases of sudden death the level of consciousness was assessed as coma but other variables were coded as 'not known'. Details of the registered cause of death and post mortem examination results were recorded in patients who died.

\section{STATISTICAL METHODS}

Standardised incidence ratios were calculated as the ratio of the observed number of district registrations to that expected by applying the total study age specific incidence rates to the relevant district populations.

Log linear modelling was used to compare the proportions of deaths in the three districts caused by subarachnoid haemorrhage, adjusting for the different age distributions.

The survival curve was estimated using the Kaplan-Meier product limit technique, survival times being censored on 30 September 1990. The combined effect of baseline prognostic variables on survival time was examined using Cox's proportional hazards regression. ${ }^{15}$ This analysis was performed separately on data for the first three weeks after the stroke and for the subsequent period. The patients' age groups and districts of residence were included in the model. Other variables were selected by a backwards stepwise procedure which excluded variables that were not significantly predictive of survival at the $5 \%$ level. The variables considered for selection were: whether living alone before the stroke, ethnic group, Rankin and Barthel scores before the stroke, coma, paralysis, incontinence, speech and swallowing impairment, and gender. For each variable any 'not known' responses were considered as a separate category.

\section{Results}

The sources of notification are given in table I. thirty two $(8 \%)$ of the cases were notified by two or more sources. Forty one $(61 \%)$ of the strokes notified by GPs were community cases which were not admitted to hospital.

Ninety four per cent of practices responded to a questionnaire sent at the end of the study and 12 extra patients were registered. Overall $78 \%$ of patients were treated in hospital, with no significant difference between the districts $\left(\chi_{2}^{2}=2 \cdot 7\right.$, $\mathrm{p}>0 \cdot 1)$.

Table II shows the number of strokes in each age group, the incidence rates per 1000 population, and the $95 \%$ confidence intervals. The standardised incidence ratio in the under $65 \mathrm{~s}$ varied from 1.48 in WL to 0.96 in LNS and 0.73 in
Table II Number of strokes (in men and women), incidence rates/ 1000 population, and 95\% confidence intervals (CI) for the incidence rates

\begin{tabular}{|c|c|c|c|c|c|c|}
\hline \multirow{2}{*}{$\begin{array}{l}\text { Age group } \\
(y)\end{array}$} & \multicolumn{3}{|c|}{ West Lambeth } & \multicolumn{3}{|c|}{ Lewisham \& $N$ Southwark } \\
\hline & No & Rate & $C I$ & No & Rate & $C I$ \\
\hline$<45$ & 11 & $0 \cdot 10$ & $0 \cdot 05,0 \cdot 18$ & 12 & 0.06 & $0 \cdot 03,0 \cdot 10$ \\
\hline $45-54$ & 9 & 0.57 & $0 \cdot 26,1 \cdot 08$ & 11 & 0.35 & $0.18,0.63$ \\
\hline & 29 & $2 \cdot 09$ & $1.40,3.00$ & 46 & 1.44 & $1.05,1.92$ \\
\hline $65-74$ & 49 & 4.09 & $3.03,5.41$ & 111 & $3 \cdot 88$ & $3 \cdot 19,4 \cdot 68$ \\
\hline Total & 98 & & & 180 & & \\
\hline
\end{tabular}

\begin{tabular}{|c|c|c|c|c|c|}
\hline \multicolumn{3}{|c|}{ Tunbridge Wells } & \multicolumn{3}{|c|}{ Total } \\
\hline$\overline{N o}$ & Rate & $C I$ & No & Rate & $C I$ \\
\hline 4 & 0.03 & $0.009,0.09$ & 27 & 0.063 & $0.04,0.09$ \\
\hline 5 & 0.21 & $0.07,0.50$ & 25 & 0.35 & $0.23,0.53$ \\
\hline 26 & $1 \cdot 20$ & $0.78,1 \cdot 76$ & 101 & 1.49 & $1 \cdot 22,1 \cdot 82$ \\
\hline 73 & $4 \cdot 03$ & $3 \cdot 16,5 \cdot 07$ & 233 & 3.97 & $3.48,4.52$ \\
\hline 108 & & & 386 & & \\
\hline
\end{tabular}


TW. There was a significant difference in incidence between the districts in those patients aged under 65 years $\left(\chi_{2}^{2}=11 \cdot 4, p<0 \cdot 01\right)$. There was no significant difference between districts in the 65-74 year old age group $(p>0 \cdot 1)$.

The mean (SD) age of patients was $64 \cdot 2(10 \cdot 1)$ years with a median of 67.5 years. Stroke patients in WL were younger (median ages: $\mathrm{WL}=65 \cdot 1$, $\mathrm{LNS}=67 \cdot 8$, and $\mathrm{TW}=68 \cdot 4$; Kruskall-Wallis test, $\mathrm{p}=0.05)$. There was an excess of men ( 218 of 386 , $(56 \%))$. Overall, $24 \%$ of subjects lived alone before the stroke. The proportion of stroke patients who were black varied between districts, WL having $20 \%$, LNS $11 \%$, and TW no black patients (table III).

Table III Distribution of cases by age, ethnic group (white (W) or black (B)), and health district (16 patients of unknown ethinic group are not included)

\begin{tabular}{|c|c|c|c|c|c|c|c|c|c|c|c|c|}
\hline \multirow[b]{2}{*}{$\operatorname{Age}(y)$} & \multicolumn{12}{|c|}{ No of patients } \\
\hline & \multicolumn{3}{|c|}{ West Lambeth } & \multicolumn{3}{|c|}{$\begin{array}{l}\text { Lewisham E } \\
N \text { Southwark }\end{array}$} & \multicolumn{2}{|c|}{$\begin{array}{l}\text { Tunbridg } \\
W \quad B\end{array}$} & $\begin{array}{l}\text { Wells } \\
(\% B)\end{array}$ & \multicolumn{2}{|c|}{${ }_{W}^{\text {All districts }}$} & $(\% B)$ \\
\hline $\begin{array}{l}<45 \\
45-54 \\
55-64 \\
65-74 \\
\text { Total }\end{array}$ & $\begin{array}{r}6 \\
4 \\
20 \\
40 \\
70\end{array}$ & $\begin{array}{r}3 \\
3 \\
7 \\
5 \\
18\end{array}$ & $\begin{array}{l}(33) \\
(43) \\
(26) \\
(11) \\
(20)\end{array}$ & $\begin{array}{r}9 \\
8 \\
39 \\
99 \\
155\end{array}$ & $\begin{array}{r}1 \\
3 \\
6 \\
9 \\
19\end{array}$ & $\begin{array}{r}(10) \\
(27) \\
(13) \\
(8) \\
(11)\end{array}$ & $\begin{array}{r}4 \\
5 \\
26 \\
73 \\
108\end{array}$ & $\begin{array}{l}0 \\
0 \\
0 \\
0 \\
0\end{array}$ & $\begin{array}{l}(0) \\
(0) \\
(0) \\
(0) \\
(0)\end{array}$ & $\begin{array}{r}19 \\
17 \\
85 \\
212 \\
333\end{array}$ & $\begin{array}{r}4 \\
6 \\
13 \\
14 \\
37\end{array}$ & $\begin{array}{r}(17) \\
(26) \\
(13) \\
(6) \\
(10)\end{array}$ \\
\hline
\end{tabular}

The Barthel and Rankin scores before stroke are given in table IV. Over $90 \%$ of patients were functionally independent before the stroke.

\section{CASE FATALITY}

During the study period, 127 (33\% of the 386 ) stroke patients died. The mean (SD) age at death was $63.5(10.5)$ years. The median length of time from stroke to death was 2.5 days in WL, 4 days in LNS, and 6.5 days in TW (Kruskal-Wallis test, $\mathrm{p}>0 \cdot 1$ ).

Thirty two patients died on the day of their stroke. The overall three week case fatality was $26 \%$ (99 out of 386) and there was no significant difference between districts for unadjusted three week case fatality $\left(\chi_{2}^{2}=0 \cdot 1, p>0 \cdot 1\right)$. There was no significant difference in case fatality between districts after standardisation for differences in age structure $\left(\chi_{2}^{2}=0 \cdot 3, p>0 \cdot 1\right)$. Twenty one $(17 \%)$ deaths were recorded as being a result of subarachnoid haemorrhage, with no difference between districts ( $p>0 \cdot 1$ ) (WL 5,LNS 11, TW 5).

Thirty per cent (38) of patients who died underwent an autopsy and a diagnosis of stroke was verified in all cases.

\section{SEVERITY INDICATORS}

Twenty five $(26 \%)$ patients in WL, $36(20 \%)$ in LNS, and $16(15 \%)$ in TW were comatose $\left(\chi_{2}^{2}=3.9, p=0 \cdot 14\right)$. Twenty one $(5 \%)$ patients were known to have had a paralysis or weakness before the stroke and $277(72 \%)$ were paralysed as

Table IV Barthel and Rankin scores before stroke (no (\%) within each district)

\begin{tabular}{|c|c|c|c|c|}
\hline Barthel disability score & $\begin{array}{l}\text { West } \\
\text { Lambeth }\end{array}$ & $\begin{array}{l}\text { Lewisham E } \\
N \text { Southwark }\end{array}$ & $\begin{array}{l}\text { Tunbridge } \\
\text { Wells }\end{array}$ & Total \\
\hline $\begin{array}{l}20 \text { Functionally independent } \\
15-19 \text { Mild disability } \\
0-14 \text { Moderate/severe disability } \\
\text { Total }\end{array}$ & $\begin{array}{rr}75 & (93) \\
5 & (6) \\
1 & (1) \\
81 & (100)\end{array}$ & $\begin{array}{rr}156 & (96) \\
5 & (3) \\
2 & (1) \\
163 & (100)\end{array}$ & $\begin{array}{rr}86 & (83) \\
13 & (13) \\
5 & (5) \\
104 & (100)\end{array}$ & $\begin{array}{rr}37 & (91) \\
23 & (7) \\
8 & (2) \\
348 & (100)\end{array}$ \\
\hline $\begin{array}{l}\text { Rankin handicap score } \\
0,1 \text {, } 2 \text { Functionally independent } \\
3 \text { Moderate } \\
4,5 \text { Moderate/severe } \\
\text { Total }\end{array}$ & $\begin{array}{rr}76 & (93) \\
5 & (6) \\
1 & (1) \\
82 & (100)\end{array}$ & $\begin{array}{rr}161 & (97) \\
5 & (3) \\
0 & (0) \\
166 & (100)\end{array}$ & $\begin{array}{r}96(91) \\
3 \quad(3) \\
6 \quad(6) \\
105(100)\end{array}$ & $\begin{array}{rr}333 & (94) \\
13 & (4) \\
7 & (2) \\
353 & (100)\end{array}$ \\
\hline
\end{tabular}

a result of the stroke. Four per cent were not paralysed and for $71(18 \%)$ (55 of whom were in a coma) assessment was not possible. Two hundred and twenty six (59\%) patients had a speech impairment, 60 of whom were in coma, and 103 $(27 \%)$ had a swallowing impairment, 58 of whom were in coma. Sixty three $(16 \%)$ patients were incontinent and $69(18 \%)$ had a urinary catheter, of whom 48 were in coma.

There was no district difference in the proportions with coma $\left(\chi_{2}^{2}=3 \cdot 9, \mathrm{p}>0 \cdot 1\right)$, paralysis $\left(\chi_{2}^{2}=0.9, \quad \mathrm{p}>0 \cdot 1\right), \quad$ swallowing impairment $\left(\chi_{2}^{2}=1 \cdot 6, \quad \mathrm{p}>0 \cdot 1\right), \quad$ or incontinence $\left(\chi_{2}^{2}=0 \cdot 9\right.$, $\mathrm{p}>0 \cdot 1)$. There was a significant difference in initial speech impairment $\left(\chi_{2}^{2}=9 \cdot 4, p=0.009\right)$ with TW having the highest proportion (WL 58\%, LNS $74 \%$, TW $78 \%$ ).

\section{SURVIVAL ANALYSIS}

The Kaplan-Meier survival curve for all patients is given in the figure.

All comatose patients were assumed to have paralysis, speech, urinary, and swallowing problems. Cox's proportional hazards regression showed significant combined effects of age, incontinence, swallowing, and coma in the first three weeks and of all these variables except incontinence in the subsequent period (table V).

\section{Discussion}

This study has used a stroke register to investigate the variation in SMR in residents aged under 65 years for three district health authority populations and has fulfilled Malmgren's criteria for a register, except for recording the diagnostic categories of stroke. ${ }^{816}$ This study was large, encompassing a population of over 600000 district residents and involving 500 GPs and 28 hospitals. No previous studies in the United Kingdom have tried to explain regional variations in mortality from stroke or have have used district health authority populations. The methodology used does not involve neurological assessment and computed tomography as part of the protocol but relies on local clinicians to make the diagnosis using investigations they consider appropriate. Comparisons with studies employing more detailed clinical methods, including computed tomograms, should therefore be made cautiously. This study did, however, use the WHO definition of stroke as recommended by Malmgren. ${ }^{8}$ Although no formal attempt to validate or standardise the diagnosis of stroke between districts was made, each case was assessed by one of the registry team who liaised with the relevant clinicians when fulfillment of the WHO criteria was in doubt. The diagnosis of stroke was verified in all cases that underwent autopsy. This methodology could be reproduced in health districts with a high SMR for stroke, including inner city districts, without the needs for protocols necessitating expensive computed tomography and detailed neurological examination, which although contributing to the diagnosis, may not be available for all patients. Although the overall population incidence cannot be assessed in this study, more meaningful agespecific stroke incidence rates can be calculated and compared with other studies. 


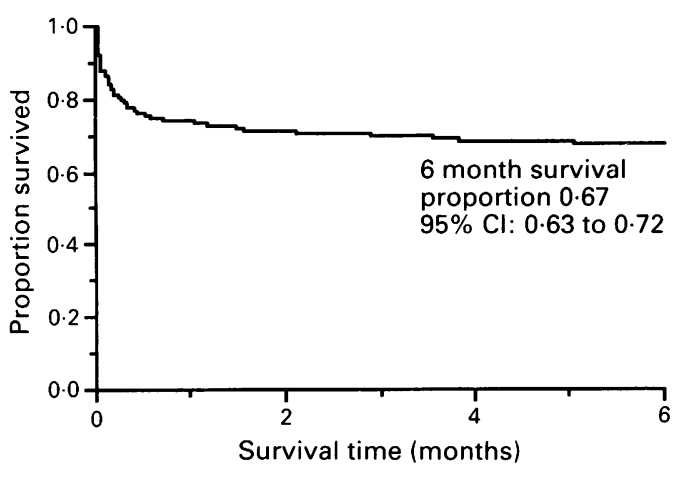

Kaplan-Meier survival curve for six months after first stroke

An initial hypothesis for this study was that the mortality rates in the under 65 year olds might be explained by differences in the incidence of stroke in the three districts. The standardised incidence ratios for first in a lifetime strokes in the under 65 s were 148 in WL, 93 in LNS, and 73 in TW, indicating significantly different incidence rates $(\mathbf{p}<0 \cdot 01)$.

This and the lack of any difference in case fatality suggests that mortality rates in this age group largely reflect the local incidence of strokes. However, these results have to be treated with due caution. We have studied only three health districts in a single region and a broader more representative sample of districts might produce different results.

In residents aged over 65 years there seems to be no significant difference in incidence between the districts. Routine data from the Office of Population Censuses and Surveys shows the age specific mortality rate for stroke in the over 65 year olds is highest in Tunbridge Wells, ${ }^{4}$ the converse to the findings in the under $65 \mathrm{~s}$.

A large proportion of strokes occurs in young blacks in the two inner city districts. This may be the result of the presence of specific risk factors for stroke in this group and particular problems in managing hypertension have been identified in African and West Indian patients in West Lambeth. ${ }^{17}$ The incidence of stroke might then be expected to increase in these districts as the black population ages. ${ }^{18}$ The results do illustrate the use of population registers to assess the prevalence of risk factors among stroke patients which could enable preventive strategies to be more effectively focused.

The incidence rates estimated from this study are lower that those quoted from some other comparable studies. Rates quoted by Malmgren, ${ }^{8}$ from studies that she regarded as technically acceptable, range from $1 \cdot 7$ to $3 \cdot 6 / 1000$ per year for $55-64$ year olds compared with 1.49 in this study, and from 4.9 to 8.9 for 65-74 year olds compared with 3.97 in this study. Many of the studies quoted by Malmgren, however, were undertaken over two decades ago and the incidence of stroke has been declining, at least in the USA. ${ }^{19}{ }^{20}$ More recent European studies quote age specific rates which are similar to those in the present study, but all studies suffer from having rates with wide confidence intervals, especially in the young age groups. ${ }^{2122}$

The Oxford stroke register was included in Malmgren's studies and was the closest in time as well as place to the current study. The study serves as a comparison with ours, although different aims, methodologies, and populations make such comparisons problematic. It also reported higher incidence rates than the present study. The Oxford study, as part of the protocol, had a higher rate of computed tomography, ${ }^{9}{ }^{16} 88 \%$ compared with $33 \%$ in the present study. It has been argued by some that computed tomograms may increase the recorded incidence of stroke but stroke is essentially a clinical diagnosis that has been shown to be reliable. ${ }^{19} 23$

The SMR for stroke in those aged under 65 years has been consistently higher in the Oxford region than in both South East Thames Regional Health Authority overall and in inner London specifically over the past decade. ${ }^{24} \mathrm{~A}$ higher incidence of stroke in Oxford would therefore be consistent with our finding that the incidence seemed to play a major role in determining the mortality from stroke. Oxford is also geographically different to the inner city and rural districts in this study and the study was based on selected general practices rather than health district populations.

If under-reporting of strokes had occurred in the present study, we might have expected those cases that were reported to have been the more severe. Though the $26 \%$ case fatality at three weeks is higher than in Oxford ${ }^{16}$ and recent studies in Italy and France, ${ }^{21} 22$ it is similar to other studies. ${ }^{25} 26$ Previous studies have not, however, published age specific case fatality rates and it is therefore not possible to compare the case fatality rate of this study of residents aged under 75 years with all age case fatality rates published previously. There was no difference in age standardised case fatality rates, and this suggests that there was no difference in case ascertainment between districts. Similarly the proportion of deaths caused by subarachnoid haemorrhage was identical in the three districts. To assess further whether the relatively low incidence rates
Table V Cox proportional hazards regression for survival after first time stroke

\begin{tabular}{|c|c|c|c|c|c|c|c|c|c|c|c|}
\hline \multirow[b]{2}{*}{ Variable } & \multirow[b]{2}{*}{ Category } & \multicolumn{5}{|c|}{ Time period: first three weeks } & \multicolumn{5}{|c|}{ Time period: three weeks onwards } \\
\hline & & $b$ & $\operatorname{se}(b)$ & $\begin{array}{l}\text { Hazard } \\
\text { ratio }\end{array}$ & $(95 \% C I)$ & p value & $b$ & $\operatorname{se}(b)$ & $\begin{array}{l}\text { Hazard } \\
\text { ratio }\end{array}$ & $(95 \% C I)$ & $p$ value \\
\hline \multirow[t]{3}{*}{ Age: } & $\leqslant 54$ & 0.00 & & 1.00 & & 0.024 & 0.00 & & 1.00 & & 0.005 \\
\hline & $55-64$ & 0.84 & 0.33 & $2 \cdot 33$ & $(1.22,4.44)$ & & 0.98 & $1 \cdot 22$ & $2 \cdot 67$ & $(0 \cdot 24,29 \cdot 16)$ & \\
\hline & $65-69$ & 0.27 & 0.36 & $1 \cdot 32$ & $(0 \cdot 66,2 \cdot 64)$ & & $1 \cdot 64$ & $1 \cdot 11$ & $\begin{array}{r}5 \cdot 16 \\
11.07\end{array}$ & $(0.59,45 \cdot 26)$ & \\
\hline \multirow[t]{4}{*}{ District: } & $\begin{array}{l}70-74 \\
\text { West }\end{array}$ & $\begin{array}{l}0.15 \\
0.00\end{array}$ & 0.36 & $\begin{array}{l}1 \cdot 16 \\
1 \cdot 00\end{array}$ & $(0.57,2.34)$ & 0.553 & $\begin{array}{l}2.40 \\
0.00\end{array}$ & & $\begin{array}{r}11.01 \\
1.00\end{array}$ & & $0 \cdot 275$ \\
\hline & Lambeth & 0.18 & 0.26 & 1.19 & $2.00)$ & & 0.68 & 0.48 & 0.51 & $(0 \cdot 20$ & \\
\hline & Southwark & 0.10 & 020 & 119 & $(0 \cdot 71,2 \cdot 00)$ & & -0.08 & 0.48 & (1) & $(0-20,100)$ & \\
\hline & $\begin{array}{l}\text { Tunbridge } \\
\text { Wells }\end{array}$ & 0.32 & $0 \cdot 30$ & $1 \cdot 38$ & $(0 \cdot 77,2 \cdot 48)$ & & $-0 \cdot 08$ & 0.49 & 0.92 & $(0.36,2 \cdot 39)$ & \\
\hline Incontinence: & $\begin{array}{l}\text { No } \\
\text { Yes. }\end{array}$ & 0.00 & & $1 \cdot 00$ & & $<0.001$ & & & & & \\
\hline \multirow{4}{*}{$\begin{array}{l}\text { Swallowing } \\
\text { impairment: } \\
\text { Coma: }\end{array}$} & $\begin{array}{l}\text { Yes: } \\
\text { No }\end{array}$ & $\begin{array}{l}1.97 \\
0.00\end{array}$ & 0.56 & $\begin{array}{l}7 \cdot 17 \\
1 \cdot 00\end{array}$ & $(2 \cdot 40,21 \cdot 47)$ & $<0.001$ & & & & & 0.008 \\
\hline & Yes & 1.61 & 0.43 & $5 \cdot 01$ & $(2 \cdot 16,11 \cdot 62)$ & & 1.03 & $0 \cdot 39$ & $2 \cdot 80$ & $(1.31,5.99)$ & \\
\hline & No & 0.00 & & 1.00 & & $<0.001$ & 0.00 & & & & 0.023 \\
\hline & Yes & $2 \cdot 19$ & $0 \cdot 29$ & $8 \cdot 91$ & $(5 \cdot 07,15.65)$ & & $1 \cdot 85$ & $0 \cdot 69$ & $6 \cdot 39$ & $(1 \cdot 65,24 \cdot 81)$ & \\
\hline
\end{tabular}

$b=$ estimated coefficient in the model, with estimated standard error se(b); hazard ratio $=e^{b}$, relative to the first-mentioned category $b=e s t i m a t e d$
of each variable; $p$ value $=$ significance of overall association of variable 
recorded in this study were the result of underreporting, we have undertaken one further study. A survey of GPs and district community rehabilitation and nursing staff at the end of the year elicited a response from $94 \%$ of practices and identified only 12 further patients, and this was after a year of intense communication with these groups.

To enable comparison of case fatality with other registers, assessment of the clinical state at the time of maximum impairment within the first 24 hours was used ${ }^{27-29}$ along with assessments of activity of daily living before the stroke. ${ }^{30}$ These and the age, living conditions before the stroke, ethnic group, and district of residence were used to predict outcome at three weeks and in the subsequent period. Proportional hazards regression analysis of survival allows the joint effects of prognostic factors on survival to be estimated.

The Rankin and Barthel scores before the stroke showed that over $90 \%$ of patients were functionally independent, which agrees with previous studies, ${ }^{30}$ but the level of disability before the stroke was not a significant independent predictor of survival.

Age had a significant effect on survival in both time periods but in the first three weeks the highest risk was to 55-64 year olds. In the subsequent period there was consistently increasing risk with age. The prediction that a comatose patient has a poor survival was consistent with other studies. ${ }^{29}{ }^{31}$ Both coma and the other indicators of severity, including incontinence and swallowing impairment were individually predictive of poor survival, especially in the first three weeks. However, district of residence and race were not significantly related to survival.

The approach taken in this study uses the minimum of resources and is readily adaptable to urban or rural areas. This study has shown that the SMRs for hypertension and stroke in three districts reflect the incidence rates and that case fatality did not contribute to the variation between districts. This information should alert public health physicians to the importance of assessing the prevalence of risk factors in their districts. The study has shown that by collecting demographic and clinical data on all incident cases of stroke in a defined population, a clear picture of the prevalence of selected risk factors in the patients can be built up. This information will allow the purchasers of health care to target, through contracts, those at highest risk of stroke. For example, in West Lambeth the young black community has a high incidence of stroke which is partly caused by uncontrolled hypertension. The results of this study shows the need for effective health education and other primary preventive strategies to reduce the incidence of hypertension and stroke in this group. Furthermore, these types of initiatives are also the focus of the British government's recent consultative white paper which aims to reduce the number of premature deaths from stroke. ${ }^{32}$

We wish to thank the Chest Heart and Stroke Association and Department of Health for funding. We are most grateful to all those who notified the register of cases, especially those in the community. Our thanks to Ana Childs for typing the manuscript.
especially the

1 Office of Population Censuses and Surveys. Mortality statistics cause. England and Wales. London: HMSO, 1988 (Series DH2, No 15)

2 Martin J, White A, Meltzer H. Office of Population Censuses and Surveys. Disabled adults: services, transport and employment (report 4, Disability in Great Britain). London: HMSO, 1989.
3 Rutstein DD, Berenberg W, Chalmers TC, Child CG, Fishman AP, Perrin EB. Measuring the quality of medical care. New Engl f Med 1976; 294: 582-8.

4 Department of Health, Office of Population Censuses and Surveys. Public Health Common Data Set 1985-1989. London: HMSO, 1990.

5 Fratiglioni L, Massey E, Schoenberg D, Schoenberg B. Mortality from cerebrovascular disease. Neuroepidemiology' 1983; 2: 101-16.

6 Charlton JRH, Hartley RM, Silver R, Holland WW. Geographical variation in mortality from conditions amenable to medical intervention in England and Wales. amenable to medical inte

7 Acheson RM, Sanderson C. Strokes: social class and geography. In: Population Trends 1978; 12: 13-17. London: HMSO

8 Malmgren R, Bamford J, Warlow C, Sandercock P. Geographical and secular trends in stroke incidence. Lancet 1987; ii: 1196-200.

9 Oxfordshire Community Stroke Project. Incidence of stroke in Oxfordshire: first year's experience of a community stroke register. BMF 1983; 287: 713-7.

10 Charlton JRH, Lakhani A, Inter Agency Comparisons Consultancy. 'Avoidable Death' indicators for DHAs 197685. London: Department of Community Medicine, UMDS, May 1988

11 Hatona S. Experience from a multicenter stroke register: a preliminary report. Bull WHO 1976; 54: 541-53.

12 Mahoney FI, Barthel DW. Functional evaluation. The Barthel Index. Maryland State Med f 1965; 14: 61-5.

13 Rankin J. Cerebral vascular accidents in patients over 65. 2. Prognosis. Scot Med f 1957; 2: 200-15.

14 Office of Population Censuses and Surveys. Monitor. London: HMSO, 1988, ppl. 88/2.

15 Dixon WJ. BMDP statistical software manual. Volume 2. Berkeley: University of California Press, 1990: 739-806.

16 Bamford J, Sandercock P, Dennis M, Burn J, Warlow C. A prospective study of acute cerebrovascular disease in the community: the Oxfordshire Community Stroke Project-1981-86. Incidence, case fatality rates and overall outcome at one year of cerebral infarction, primary intracerebral and at one year of cerebral infarction, primary intracerebral and
subarachnoid haemorrhage. $\mathcal{F}$ Neurol Neurosurg Psychiatry 1990; 53: 16-22.

17 Morgan M, Watkins CJ. Managing hypertension: Beliefs and responses to medication among cultural groups. Foumal of Social Health \& Illness 1988; 10: 561 -78.

8 Hegman A, Karp HR, Heyden S, Bartel A, Cassel JC, Tyroler HA, Comini J, Hames CG, Stuart W. Cerebrovascular disease in the bi-racial population of Evans County, Georgia. Stroke 1971; 2: 509-17.

19 Garraway W, Whisnant J, Drury I. The continuing decline in the incidence of stroke. Mayo Clin Proc 1983; 58: 520-3.

20 Broderick JP, Phillips SJ, Whisnant JP, O'Fallon WM Stroke incidence in Rochester Minnesota, 1945-84. The Stroke incidence in Rochester Minnesota, 1945-84. The
end of the decline in stroke? Neurologv 1988; 38 (Suppl) 146.

21 Ricci S, Grazia M, La Rosa F, Vitali R et al. SEPIVAC: community-based study of stroke incidence in Umbria,
Italy. $\mathcal{F}$ Neurol Neurosurg Psychiatry 1991: 54: 695-8.

22 Giroud M, Milan C, Beuriat P, Gras P, Essayagh E, Arveux $\mathrm{P}$, Dumas R. Incidence and survival rates during a two-year period of intracerebral and subarachnoid haemorrhages, cortical infarcts, lacunes and transient ischaemic attacks. The stroke registry of Dijon. 1985-1989. Int 7 Efidemiol 1991; 20: 892-9

23 Sandercock P, Molyneux A, Warlow C. Value of computed tomograph in patients with stroke: The Oxfordshire Community Stroke Project. BMF 1985; 290: 193-7.

24 Office of Population Censuses and Surveys. Mortality statistics. Area: England and Wales. London: HMSO 198188 (Series DH5).

25 Matsumo N, Whisnant JP, Kurland LT, Okazaki H. Natural history of stroke in Rochester, Minnesota, 1955 through 1969: an extension of a previous study, 1945 through 1954 Stroke 1973; 4: 20-9.

26 Gross CR, Kase CS, Mohr JP, Cunningham SC, Baker WE Stroke in South Alabama: incidence and diagnostic features-a population based study. Stroke 1984; 15 $249-55$

27 Aho K, Harmsen P, Hatano S, Marquardsen J, Smirnov VE, Strasser $T$. Cerebrovascular disease in the community: result of the WHO collaborative study. Bull WHO 1980 ; 8(i): $113-30$.

28 Kuller L, Anderson PHH, Peterson D, Cassel J, Spiers P, Curry H. National cerebrovascular disease morbidity study. Stroke 1970; i: 86-99.

29 Ahmed OI, Orchard TI, Sharma R, Mitchell H, Talbot E. Declining mortality from stroke in Allegheny County, Pennsylvania, trends in case fatality and severity of disease, 1971-1980. Stroke 1988; 19: 181--4

Malmgren R, Bamford J, Warlow C, Sandercock P, Slattery $J$. Projecting the number of patients with first ever strokes and patients newly handicapped by stroke in England and Wales. BMF 1989; 298: 656-60

31 Howard G, Walker M, Becker C, Coull B, Feibel J, Mcleroy K, Toole JF, Yatsu F. Community hospital based stroke programs: North Carolina, Oregon and New York. iii. Factors influencing survival after stroke: proportional hazards analysis of 4219 patients. Stroke 1986; 17(2): 294-9.

32 Secretary of State for Health. The health of the nation: a consultative document for health in England. London: HMSO, 1991, (CM1523). 